\title{
Discovering frequency sensitive thalamic nuclei from EEG microstate informed resting state fMRI
}

\author{
Simon Schwab, ${ }^{1,2, *}$ Thomas Koenig, ${ }^{1,2}$ Yosuke Morishima, ${ }^{1,2,3}$ Thomas Dierks, ${ }^{1,2}$ Andrea Federspiel, $, 1,2$ \\ Kay Jann ${ }^{4}$ \\ ${ }^{1}$ Department of Psychiatric Neurophysiology, University Hospital of Psychiatry, and University of Bern, Bern, \\ Switzerland \\ ${ }^{2}$ Center for Cognition, Learning and Memory, University of Bern, Bern, Switzerland \\ ${ }^{3}$ Japan Science and Technology Agency, PRESTO, 4-1-8 Honcho Kawaguchi, Saitama 332-0012, Japan \\ ${ }^{4}$ Department of Neurology, University of California, Los Angeles, California, United States \\ * Corresponding author: Simon Schwab, PhD, Department of Psychiatric Neurophysiology, University Hospital \\ of Psychiatry, Bolligenstrasse 111, 3000 Bern 60, Switzerland \\ Phone: +41 31930 9396; E-mail: schwab@puk.unibe.ch
}

Running title: Frequency sensitive thalamic nuclei from EEG-fMRI 


\section{Abstract}

Microstates (MS), the fingerprints of the momentarily and time-varying states of the brain derived from electroencephalography (EEG), are associated with the resting state networks (RSNs). However, using MS fluctuations along different EEG frequency bands to model the functional MRI (fMRI) signal has not been investigated so far, or elucidated the role of the thalamus as a fundamental gateway and a putative key structure in cortical functional networks. Therefore, in the current study, we used MS predictors in standard frequency bands to predict blood oxygenation level dependent (BOLD) signal fluctuations. We discovered that multivariate modeling of BOLD-fMRI using six EEG-MS classes in eight frequency bands strongly correlated with thalamic areas and large-scale cortical networks. Thalamic nuclei exhibited distinct patterns of correlations for individual MS that were associated with specific EEG frequency bands. Anterior and ventral thalamic nuclei were sensitive to the beta frequency band, medial nuclei were sensitive to both alpha and beta frequency bands, and posterior nuclei such as the pulvinar were sensitive to delta and theta frequency bands. These results demonstrate that EEG-MS informed fMRI can elucidate thalamic activity not directly observable by EEG, which may be highly relevant to understand the rapid formation of thalamocortical networks.

Key words: EEG microstates, EEG topography, thalamus, fMRI, resting-state 


\section{Introduction}

The past decades have revealed the complex structural and functional organization of the brain. Organized functional networks are not only dominant during a task, but also during a "baseline" state, when participants are at rest and not involved in any specific task or behavior (Fox and Raichle 2007). For example, analyses of blood oxygenation level-dependent (BOLD) signal fluctuations at rest reveal regions in the primary motor and supplementary motor areas similar to those active during a finger tapping task (Biswal et al. 1995). Multiple so called functional resting state networks (RSNs) have been identified, such as visual, default mode, memory, motor, sensorimotor, and executive control networks (Damoiseaux et al. 2006; Fransson et al. 2007; Raichle et al. 2001; Smith et al. 2009). These cortical networks further possess projections to thalamic nuclei, with their fundamental role as gateways of motor and sensory information from the body to distributed zones of the cerebral cortex. Furthermore, the thalamo-cortical mapping of networks has been established using probabilistic tractography (Behrens et al. 2003; Klein et al. 2010) as well as fMRI functional connectivity (Zhang et al. 2008 2010). That the thalamus is also involved in higher order cognition, as well as cortico-cortical communication has been suggested based on the physiology and histology of the thalamus (Sherman 2007). Besides MRI techniques, electroencephalography (EEG) research has suggested the thalamus as a potential mediator of the cortical EEG signal through computational models and empirical data (Lopes da Silva 1991; Lopes da Silva et al. 1973 1980). For example the pulvinar and lateral geniculate nucleus contribute to alpha rhythms and thalamocortical coherence (Lopes da Silva et al. 1973 1980). Hence, even though thalamic activity is not directly measured by EEG, the thalamus nevertheless is closely connected to the neocortex and is able to modulate cortical activity.

While studies employing simultaneous electroencephalography (EEG) and fMRI provide strong evidence for a neurophysiologic coupling between cortical networks (RSNs) and EEG scalp field potentials and frequency oscillations (Britz et al. 2010; Jann et al. 2010; Mantini et al. 2007), none of these studies explored the thalamic involvement in cortical EEG characteristics. However, as outlined above, both cortical EEG oscillations and fMRI signal fluctuations involve thalamic modulation. Here we postulate that EEG informed fMRI would be a sensitive method for detecting thalamic activity (Schwab et al. 2013). Thus, the present study was designed to extend previous research using a novel analysis approach that accounts for EEG frequency band oscillations as well as their topographic scalp field distribution to predict thalamic BOLD signal fluctuations.

EEG frequency bands provide valuable information regarding the participants' vigilance and mental states. 
For example, different stages of consciousness are associated with specific frequency bands, such as: stage 2 sleep contains theta waves, stage 3 sleep contains delta waves, alpha waves are associated with inattention, and beta waves are associated with cognitive operations (Laufs et al. 2003). Recently, cortical maps of spectral EEG signatures during spontaneous fMRI signals were created and used to determine clusters of brain areas that represent prominent functional subdivisions (Liu et al. 2014).

On the other hand, and besides frequency, cluster analyses can extract global topographic scalp field distributions across all electrodes (Lehmann et al. 1987). A small number of topographic distributions are sufficient to explain large portions of the EEG activity ( $>80 \%$ of total variance) with high reproducibility, even in studies with large samples (Koenig et al. 2002 2005b). These topographies are termed microstates (MS), as they represent momentarily stable distributions of the scalp electric potentials with durations of tens to a few hundred milliseconds. As such, MS are conceptualized as fingerprints of the momentarily and time-varying states of the brain. The dynamics of these MS may be the basis for rapid reorganization and adaptation of functional brain networks (Van de Ville et al. 2010). Additionally, differences in MS occurrence and duration may be important to altered brain function in psychiatric conditions such as panic disorder (Kikuchi et al. 2011), dementia (Nishida et al. 2013; Strik et al. 1997), schizophrenia (Andreou et al. 2014; Kindler et al. 2011; Koenig et al. 1999; Lehmann et al. 2005), and Tourette syndrome (Stevens et al. 1996).

Therefore, in this study, we employ MS topography specific frequency decomposition of the EEG oscillations, since both the different standard EEG frequency bands as well as scalp field distribution, such as MS, are relevant for functional network analyses. We expect this strategy to elucidate prominent cortical and subcortical functional areas associated with EEG-MS classes, specifically including those located in the thalamus.

\section{Materials and Methods}

\section{Participants}

We reanalyzed a subset of the data from Jann et al. (2010). Fourteen healthy university students (six female, eight male, median age 27 years, range: 22-31 years) underwent simultaneous EEG-fMRI during a resting state, with eyes closed but not falling asleep. They were instructed to relax and not think about anything in particular. All data collection occurred in the morning and participants did not have caffeine, nicotine, or alcohol for at least $10 \mathrm{~h}$ prior to the experiment. Study exclusion criteria included contraindications against use of MRI, a history of neurological or psychiatric conditions, and use of psychoactive medication or illegal drugs. 


\section{Ethics Statement}

This study was approved by the ethics committee of Kantonale Ethikkommission, Bern, Switzerland. Written informed consent was obtained from all participants prior to their participation, in accordance with the tenets of the Declaration of Helsinki. Data has been fully anonymized.

\section{MRI data acquisition}

We acquired MRI data from a 3T Siemens Magnetom Trio, and functional data with an EPI sequence (252 volumes, 32 slices, $3 \times 3 \times 3 \mathrm{~mm}^{3}$, gap thickness $0.75 \mathrm{~mm}$, matrix size $64 \times 64$, FOV $192 \times 192 \mathrm{~mm}^{2}$, TR $1980 \mathrm{~ms}$, TE 30 ms. We recorded structural images after EEG-fMRI acquisition and removal of the EEG cap using a MDEFT sequence (176 sagittal slices, $1.0 \mathrm{~mm}$ slice thickness, FOV 256×256 mm², TR $7.92 \mathrm{~ms}$, TE $2.48 \mathrm{~ms}$ ).

\section{EEG data acquisition}

We acquired EEG data with a 96 channel MR compatible system (Brain Products, Germany). The input range was $16.3 \mathrm{mV}$, with a resolution of $0.25 \mu \mathrm{V}$. Two electrodes below the clavicles recorded the electrocardiogram (ECG), and another two electrodes below the eye recorded an electrooculogram (EOG). Each MR scan volume was automatically marked in the EEG data. The EEG was bandpass filtered between $0.1 \mathrm{~Hz}$ and $250 \mathrm{~Hz}$ and sampled with $5 \mathrm{kHz}$. Impedance between the electrodes and the participant's scalp were kept below $50 \mathrm{k} \Omega$.

\section{fMRI data preprocessing}

Preprocessing using SPM8 (http://www.fil.ion.ucl.ac.uk/spm) included slice time and motion correction, co-registration to anatomical data, normalization to MNI space $(2 \times 2 \times 2 \mathrm{~mm})$, and spatial smoothing $(\mathrm{FWHM}=8 \mathrm{~mm})$.

\section{EEG data preprocessing}

EEG preprocessing used BrainVision Analyzer (version 1.05.0005, Brain Products, Germany) and included scan pulse artifact correction by average artifact subtraction (AAS) (Allen et al. 1998) and cardioballistic artifact correction (CBA) by independent component analysis (ICA) (Jann et al. 2009 2008). EEG time courses were downsampled to $100 \mathrm{~Hz}$ and bandpass filtered $(1-30 \mathrm{~Hz})$. Epochs exhibiting residual artifacts (eyes, muscles, etc.) were removed. The cleaned EEG was then subjected to a Topographic Time-Frequency 
Decomposition (Koenig et al. 2001b). For this purpose, the multichannel EEG data was transformed into the time-frequency domain using complex Gabor-wavelets. The real and complex parts of the resulting topography vectors of wavelet coefficients were then submitted to a modified k-means clustering algorithm (Pascual-Marqui et al. 1995), yielding six prototypical map configurations per participant. Next, these individual maps were averaged across participants into mean MS classes using a permutation procedure (Koenig et al. 1999). Each topography vector of all EEG wavelet coefficients was then assigned to the best fitting mean MS class, and the time-frequency dynamics of each MS class was reconstructed based on this assignment. Finally, these dynamics were collapsed into eight standard EEG frequency bands (Jann et al. 2010): $1.0 \mathrm{~Hz}<\delta \leq 3.5 \mathrm{~Hz}, 3.5 \mathrm{~Hz}<$ $\theta_{1} \leq 6.25 \mathrm{~Hz}, 6.25 \mathrm{~Hz}<\theta_{2} \leq 8.2 \mathrm{~Hz}, 8.2 \mathrm{~Hz}<\alpha_{1} \leq 10.5 \mathrm{~Hz}, 10.5 \mathrm{~Hz}<\alpha_{2} \leq 14.0 \mathrm{~Hz}, 14.0 \mathrm{~Hz}<\beta_{1} \leq$ $18.75 \mathrm{~Hz}, 18.75 \mathrm{~Hz}<\beta_{2} \leq 21.88 \mathrm{~Hz}, 21.88 \mathrm{~Hz}<\beta_{3} \leq 30.0 \mathrm{~Hz}$.

We investigated variability of the MS classes among subjects, and spatially correlated the six mean classes with the subjects' six individual classes. The spatial correlation for the mean class A was .84, class B .90, class C .75, class D .91, class E .89, and class F .81; the mean MS classes are shown in Fig. 3. In average, the mean classes explained $73 \%$ of the variance in the subjects' classes.

\section{Microstate informed fMRI analysis}

Instead of downsampling MS time courses to match fMRI timecourse, each MS time point reflects a "summary statistic", i.e., the average power over the whole TR per frequency band, and for the individual MS classes, and thus corresponds to the fMRI acquisition time points, see Fig. 1. We applied linear interpolation to segments of low EEG data quality, which affected $5.7 \pm 3.4 \%$ of the data points in 13 participants; one participant was not affected by segments of lower EEG data quality. We removed the first six and last eight data points, because here data was extrapolated, leaving 238 data points remaining. Data was normalized along frequency bands, and convolved with the canonical hemodynamic response function. The resulting MS regressors (14 participants $\times 8 \mathrm{MS}$ frequency bands $\times 6 \mathrm{MS}$ classes) were subsequently used to predict BOLD-fMRI responses in each participant. For each of the 48 MS regressors, a general linear model (GLM) was used to model each participants's BOLD activity. We also implemented regressors of non interest such as six movement parameters and their first derivative, a cerebrospinal fluid and white matter seed (Fox et al. 2009), and multiple binary regressors according to the number of interpolated low quality EEG segments for each participant. Using the resulting $48 \beta$-maps from each participant, we conducted voxel wise statistical analyses in R (http://www.rproject.org/). A random effects analysis was conducted (8 MS frequency bands $\times 6$ MS voxel wise ANOVA; 
Fig. 2), and for each MS class, a one-way ANOVA was also conducted. Fig. 3 shows brain areas related to changes in MS frequency band. All maps were masked with a joint mask (logical OR) of the gray matter (GM) image from segmentation (GM probability > .20) and a thalamus mask from the "WFU PickAtlas" (Maldjian et al. 2003) to ensure that the thalamus was fully included. Maps were FDR corrected for multiple comparison (Genovese et al. 2002), except Fig. 2C, which is uncorrected $(p<.001)$. Statistical maps were overlayed onto the "ch2bet" average T1 weighted image from MRICron. We extracted eight thalamic nuclei from the "WFU PickAtlas" (Maldjian et al. 2003) to calculate percentage coverage of our MS related activity with specific nuclei. The eight nuclei had a mean voxel size of $84 \mathrm{vx}\left(1 \mathrm{vx}\right.$ is $\left.2 \times 2 \times 2 \mathrm{~mm}^{3}\right)$, the smallest were the lateral dorsal n. (12 vx) and the ventral posterior medial n. (17 vx), the largest was the pulvinar with $267 \mathrm{vx}$. The sorted exact ROI sizes of the eight nuclei were: $12 \mathrm{vx}, 17 \mathrm{vx}, 30 \mathrm{vx}, 42 \mathrm{vx}, 72 \mathrm{vx}, 111 \mathrm{vx}, 122 \mathrm{vx}$, and $267 \mathrm{vx}$ (average voxel size between the left and contralateral right nucleus). For the registration of the anatomical images with the thalamus atlas, see Inline Supplementary Figure 1, for the registration with the BOLD images, see Inline Supplementary Figure 2.

\section{Frequency color coding}

The maps in Fig. 3 were created from FDR corrected $F$-maps based on results of one-way ANOVAs for the effects of frequency. However, since we investigated eight frequency bands, significant $F$-values could result from a variety of differences in means between the eight frequency bands that are not visible in statistical $F$ maps. For example, higher beta estimates among the high frequencies compared with lower beta estimates among the middle and low frequencies, and vice versa, may produce this result. Therefore, for each significant thalamic voxel, we calculated mean beta estimates along three groups of frequency bands: low $\left(\delta, \theta_{1}, \theta_{2}\right)$, medium $\left(\alpha_{1}, \alpha_{2}\right)$, and high $\left(\beta_{1}, \beta_{2}, \beta_{3}\right)$. This grouping allowed us to perform linear transformations of the beta estimates to RGB values, with red coding for low, green for middle, and blue for high frequencies. Since most beta estimates in the thalamus were positive (92\%), only these positive estimates were transferred to RGB color values. Color coding was based on the frequency group responsible for the significant main effect. For example, yellow reflects high beta estimates in the low (red) and middle (green) frequency band, and lower beta estimates in the high frequency band (blue). Thus, RGB color values represent a wide range of possible differences in the mean among these three frequency groups. 


\section{Results}

We analyzed BOLD estimates from the MS informed fMRI analyses. BOLD estimates were modeled by six MS classes and eight frequency bands in a random effects model, depicting brain regions significantly associated with changes in MS classes, changes in frequency band, or both (Fig. 2). The resulting maps indicated that areas of significant change exist in distributed cortical areas and also in subcortical structures, especially the thalamus. A significant statistical interaction between MS classes and frequency bands contributed to explaining BOLD changes in frontal, temporal, parietal and occipital areas, and also in the thalamus (see Fig. 2A). Additionally, the main effect of frequency band information revealed large significant areas in all four major cortical lobes and in the thalamus (Fig. 2B). MS class changes were reflected only in the thalamus and small cortical areas in the right frontal cortex and left postcentral gyrus (Fig. 2C). All three maps A-C (Fig. 2) revealed clusters in the thalamus (MS class $\times$ frequency band: 174 voxels, peak $F=3.46,4-1812 \mathrm{~mm}$; frequency band: 736 voxels, peak $F=16.8,2-2010 \mathrm{~mm}$; MS class: 23 voxels, peak $F=5.98,4-1614 \mathrm{~mm}$ ).

Due to the interaction effect for thalamic areas (Fig. 2A), we expected further analyses to reveal characteristic thalamic frequency effect patterns for different MS classes. Thus, we performed one-way ANOVAs on the main effect frequency band (eight standard EEG frequency bands) separately for each of the six MS classes, resulting in a statistical map for each specific MS class. Fig. $3 \mathrm{~A}-\mathrm{F}$ shows the thalamic main effects of frequency band for each MS class. We found that in the MS classes B, C, D, and F the ventral thalamic structures were associated with a frequency band effect in the high frequency group (blue), while in the medial dorsal nucleus (MD) the effect occurred for both the high and medium frequencies (cyan). In the ventral pulvinar, the frequency band effect occurred for low frequencies (see MS classes D and F at $2 \mathrm{~mm}$ ), whereas in the dorsal pulvinar the frequency effect occurred for high frequencies (MS classes $B, C$, and $F$ at $6 / 10 \mathrm{~mm}$ ). In Map $E$, the frequency band effect in the right MD occurred for medium frequencies (green), and in the right VPL the effect occurred for low frequencies (red).

Altogether, the strongest thalamic activity occurred for class F, as can be seen in bar plots for the individual thalamic nuclei and by the values for percent of significant voxels (Fig. 3). There were no effects in the thalamus for MS class A.

We investigated the correlation and shared information among the 48 regressors involved in the microstate informed fMRI analysis (before applying HRF convolution), see Fig. 4. Adjacent regressors within the same microstate class were correlated. Since the resulting beta maps from correlated regressors are almost identical, 
we consider the results from the ANOVA analysis to reflect systematic changes in beta values from uncorrelated regressors. Using fewer frequency bands (we chose eight) would probably be still sufficient to reproduce the results we presented here. On the other hand, these 8 standard frequency bands represent a very common subdivision according to the EEG literature.

\section{Discussion}

There are a few previous studies that conducted MS informed fMRI (Britz et al. 2010; Musso et al. 2010; Yuan et al. 2012), and established associations between MS and the cortical RSNs, but none of these investigated involvement of the thalamus. Therefore, we implemented EEG-MS informed fMRI analyses to discover associated BOLD-fMRI functional thalamic activity.

The main findings of the present study were strong thalamic localization by MS informed fMRI, as well as evidence for frequency-specific activation patterns of various thalamic nuclei for individual MS. The data revealed specific thalamic patterns for MS classes. Non-invasive thalamocortical mapping has already been

established in probabilistic tractography (Behrens et al. 2003; Klein et al. 2010) and fMRI functional connectivity (Zhang et al. 2008 2010). Significant contributions of thalamic nuclei are feasible because the thalamus has a fundamental role as a gateway of motor and sensory information to distributed zones of the cerebral cortex, and as a potential mediator of the cortical EEG signal (Lopes da Silva 1991; Lopes da Silva et al. 1973 1980). In this context, it is important to note that the procedure used to derive each predictor for thalamic activation explicitly modeled the scalp EEG with a single phase angle across all electrodes (Koenig et al. 2005b). Common phase oscillations in distributed cortical networks have received theoretical interest (Tononi and Koch 2008) and have empirically been shown to be important in cognition (Koenig et al. 2001a 2005a; Kottlow et al. 2012). Furthermore, brain oscillations also adapt to external rhythms, allowing the brain to optimally process predictable events, such as speech (Calderone et al. 2014). The present data therefore suggests that the thalamus is important not only for the modulation of the amplitude of cortical oscillations, but also for the establishment of the synchrony of these oscillations among different cortical regions.

Our data indicate that while most MS exhibited thalamic activity, two maps had little or no thalamic response (A and E). Specific MS may involve corticothalamic functional networks, but other cortico-cortical networks with minimal or no thalamic involvement may exist. Networks involving the thalamus may be associated with primary cortical areas, due to the fact that large parts of the thalamus are directly connected 
to these areas (e.g., most of the ventral nuclei: VL, VPL, and VPM). This explanation is consistent with the fact that some MS are assigned to primary cortical networks (sensory, visual), whereas others are assigned to higher cognitive networks (DMN, attention). Thus, it is highly plausible that specific MS involve different thalamic nuclei.

VA, VL, and MD thalamic nuclei were coactivated with several MS, and all three nuclei were involved in four of the six MS classes (B, C, D, and F). VA and VL were connected to premotor and motor cortex, respectively. The motor network has been assigned to multiple MS classes (Yuan et al. 2012) (class B was similar to Yuan et al.'s MS11, C to MS10, D to MS7, and F to MS4). Conversely, the MD nucleus connects to frontal areas and has a key role in attention, among other frontal lobe functions. We found the largest MD nucleus coverage in classes $\mathrm{C}$ and $\mathrm{F}$. Our class $\mathrm{C}$ categorization most likely corresponds to Britz et al.'s MS class $\mathrm{C}$, which was assigned to the frontal network. Our class $\mathrm{F}$ categorization corresponds to Yuan et al.'s class MS4, which is assigned to the attention network. In addition, these two classes (C, F) were the only classes showing LP coverage. LP is a key component of the neural circuitry for directed attention (Kamishina et al. 2009) and LP has direct connections to the posterior parietal cortex; damage to this area results in information neglect (Reep and Corwin 2009). Thus, in the context of the well known cortical projections of the thalamic nuclei to the different cortical regions, the thalamic patterns detected in the presented study are largely consistent with the results from previous studies that related the MS to the different RSNs. In the present study, we indirectly investigated (compared to neurophysiology animal studies) frequency tuning of the human thalamus. We used MS time series in the eight standard EEG frequency bands to predict the BOLD signal using a multivariate approach, in order to create a statistically derived map of thalamic frequency sensitive areas. RGB colors coded for three frequency groups (low, medium, and high frequency bands) to identify which bandwidth or combination of bandwidths drove the statistical effects. Most areas were tuned to both alpha and beta (medial thalamus), or beta (ventral thalamus). The alpha band was often associated with inactivity and the DMN: For example, increased posterior alpha power was correlated with decreased MRI signal in occipital, superior temporal, inferior frontal, and cingulate cortex, and with increased signal in the thalamus and insula (Goldman et al. 2002). Our results demonstrate that the MD nucleus most prominently exhibited alpha and beta tuning. One explanation for these findings is that the MD nucleus is a key thalamic area for connections to the frontal cortex when people are at rest and engaged in self-reflection. This thalamocortical relationship may also be relevant to clinical conditions in which the DMN and/or EEG alpha are impaired, such as in schizophrenia, Alzheimer's disease, and autism (Buckner et al. 2008; Greicius et al. 2004; Kennedy et al. 
2006; Whitfield-Gabrieli et al. 2009; Zhou et al. 2007).

Our data demonstrate beta tuning in the ventral areas of the thalamus (VL, VPL); this part of the thalamus is known to project to the sensorimotor cortex. Indeed, beta rhythms are associated with activation of the primary somatosensory and motor cortices (Liu et al. 2014; Ritter et al. 2009). We found delta/theta frequency tuning in the pulvinar, as visualized in map D and F. This finding contrasts with the finding that the pulvinar can act as a posterior alpha generator (Liu et al. 2012). An explanation for this discrepancy is that the pulvinar, which is the largest substructure in the thalamus, can be further subdivided based on function. The pulvinar also projects to most major cortical regions (occipital, temporal, and parietal areas). Indeed, our results demonstrate not only an association between low frequencies and dorsal parts of the pulvinar (F: $2 \mathrm{~mm}$ ), but also between high frequencies and lateral inferior parts of the pulvinar ( $F$ : 10-14 mm), but an even finer subdivision of the pulvinar would reach the limits of our fMRI data resolution.

While our results demonstrate strong thalamic involvement of specific substructures that relate to neural cortical oscillations, some limitations need to be addressed comparing our results with previous studies. First, there are differences between studies in the complexity and diversity of analyses. For example, while previous studies (Britz et al. 2010; Yuan et al. 2012) usually correlated BOLD with MS time series that were convolved and subsequently downsampled, our approach implemented a "summary statistic" of the whole TR to address the problem of undersampling. Also, using time-frequency analysis, we also integrate frequency information beside topographic data, and subsequently used both of these properties to model BOLD fluctuations. This approach constitutes a more sensitive approach and extends previous research.

\section{Conclusions}

Thalamic involvement in MS informed fMRI has not always been evident or conclusive. The present findings demonstrate that cortical EEG-MS properties were associated with the thalamus, and could be used to map thalamic frequency tuning. We discovered functionally involved substructures in the thalamus associated with the EEG signals, and we identified their specific frequency tuning for various MS. Further research is needed in order to evaluate thalamocortical networks related to EEG-MS fluctuation. Establishing thalamocortical functional relationships may have important implications for the further understanding of RSNs and may also exhibit clinical relevance (Greicius et al. 2007; Nair et al. 2013). 


\section{Acknowledgments}

This research was supported in part by two grants from the Swiss National Science Foundation (SNSF) to Thomas Koenig (Sinergia \#136249), and Kay Jann (SPUM \#33CM30-124114).

\section{Author Contributions}

Experimental conception and design: K.J., T.K., T.D. Data analyses: S.S., T.K., K.J., Y.M. Contribution of analysis tools: S.S., T.K. Writing of first draft of the paper: S.S. All authors contributed to and have approved the final manuscript. 


\section{References}

Allen, P.J., Polizzi, G., Krakow, K., Fish, D.R., Lemieux, L. 1998. Identification of EEG events in the MR scanner: the problem of pulse artifact and a method for its subtraction. Neurolmage 8(3), 229-239. doi:10.1006/nimg.1998.0361.

Andreou, C., Faber, P.L., Leicht, G., Schoettle, D., Polomac, N., Hanganu-Opatz, I.L., Lehmann, D., Mulert, C. 2014. Resting-state connectivity in the prodromal phase of schizophrenia: insights from EEG microstates. Schizophr. Res. 152(2-3), 513-520. doi:10.1016/j.schres.2013.12.008.

Behrens, T.E.J., Johansen-Berg, H., Woolrich, M.W., Smith, S.M., Wheeler-Kingshott, C.A.M., Boulby, P.A., Barker, G.J., Sillery, E.L., Sheehan, K., Ciccarelli, O., Thompson, A.J., Brady, J.M., Matthews, P.M. 2003. Non-invasive mapping of connections between human thalamus and cortex using diffusion imaging. Nat. Neurosci. 6(7), 750-757. doi:10.1038/nn1075.

Biswal, B., Yetkin, F.Z., Haughton, V.M., Hyde, J.S. 1995. Functional connectivity in the motor cortex of resting human brain using echo-planar MRI. Magn. Reson. Med. 34(4), 537-541.

Britz, J., Van De Ville, D., Michel, C.M. 2010. BOLD correlates of EEG topography reveal rapid resting-state network dynamics. Neurolmage 52(4), 1162-1170. doi:10.1016/j.neuroimage.2010.02.052.

Buckner, R.L., Andrews-Hanna, J.R., Schacter, D.L. 2008. The brain's default network: anatomy, function, and relevance to disease. Ann. N. Y. Acad. Sci. 1124, 1-38. doi:10.1196/annals.1440.011.

Calderone, D.J., Lakatos, P., Butler, P.D., Castellanos, F.X. 2014. Entrainment of neural oscillations as a modifiable substrate of attention. Trends Cogn. Sci. 18(6), 300-309. doi:10.1016/j.tics.2014.02.005.

Damoiseaux, J.S., Rombouts, S.A.R.B., Barkhof, F., Scheltens, P., Stam, C.J., Smith, S.M., Beckmann, C.F. 2006. Consistent resting-state networks across healthy subjects. Proc. Natl. Acad. Sci. U. S. A. 103(37), 13848-13853. doi:10.1073/pnas.0601417103.

Fox, M.D., Raichle, M.E. 2007. Spontaneous fluctuations in brain activity observed with functional magnetic resonance imaging. Nat. Rev. Neurosci. 8(9), 700-711. doi:10.1038/nrn2201.

Fox, M.D., Zhang, D., Snyder, A.Z., Raichle, M.E. 2009. The global signal and observed anticorrelated resting state brain networks. J. Neurophysiol. 101(6), 3270-3283. doi:10.1152/jn.90777.2008. 
Fransson, P., Skiöld, B., Horsch, S., Nordell, A., Blennow, M., Lagercrantz, H., Aden, U. 2007. Restingstate networks in the infant brain. Proc. Natl. Acad. Sci. U. S. A. 104(39), 15531-15536. doi: $10.1073 /$ pnas. 0704380104 .

Genovese, C.R., Lazar, N.A., Nichols, T. 2002. Thresholding of statistical maps in functional neuroimaging using the false discovery rate. Neurolmage 15(4), 870-878. doi:10.1006/nimg.2001.1037.

Goldman, R.I., Stern, J.M., Engel, Jr, J., Cohen, M.S. 2002. Simultaneous EEG and fMRI of the alpha rhythm. Neuroreport 13(18), 2487-2492. doi:10.1097/01.wnr.0000047685.08940.d0.

Greicius, M.D., Flores, B.H., Menon, V., Glover, G.H., Solvason, H.B., Kenna, H., Reiss, A.L., Schatzberg, A.F. 2007. Resting-state functional connectivity in major depression: abnormally increased contributions from subgenual cingulate cortex and thalamus. Biol. Psychiatry 62(5), 429-437. doi:10.1016/j.biopsych.2006.09.020.

Greicius, M.D., Srivastava, G., Reiss, A.L., Menon, V. 2004. Default-mode network activity distinguishes Alzheimer's disease from healthy aging: evidence from functional MRI. Proc. Natl. Acad. Sci. U. S. A. 101(13), 4637-4642. doi:10.1073/pnas.0308627101.

Jann, K., Dierks, T., Boesch, C., Kottlow, M., Strik, W., Koenig, T. 2009. BOLD correlates of EEG alpha phase-locking and the fMRI default mode network. Neurolmage 45(3), 903-916.

Jann, K., Kottlow, M., Dierks, T., Boesch, C., Koenig, T. 2010. Topographic electrophysiological signatures of FMRI Resting State Networks. PLoS One 5(9), e12945. doi:10.1371/journal.pone.0012945.

Jann, K., Wiest, R., Hauf, M., Meyer, K., Boesch, C., Mathis, J., Schroth, G., Dierks, T., Koenig, T. 2008. BOLD correlates of continuously fluctuating epileptic activity isolated by independent component analysis. Neurolmage 42(2), 635-648. doi:10.1016/j.neuroimage.2008.05.001.

Kamishina, H., Conte, W.L., Patel, S.S., Tai, R.J., Corwin, J.V., Reep, R.L. 2009. Cortical connections of the rat lateral posterior thalamic nucleus. Brain Res. 1264, 39-56. doi:10.1016/j.brainres.2009.01.024.

Kennedy, D.P., Redcay, E., Courchesne, E. 2006. Failing to deactivate: resting functional abnormalities in autism. Proc. Natl. Acad. Sci. U. S. A. 103(21), 8275-8280. doi:10.1073/pnas.0600674103.

Kikuchi, M., Koenig, T., Munesue, T., Hanaoka, A., Strik, W., Dierks, T., Koshino, Y., Minabe, Y. 2011. EEG microstate analysis in drug-naive patients with panic disorder. PLoS One 6(7), e22912. doi:10.1371/journal.pone.0022912. 
Kindler, J., Hubl, D., Strik, W.K., Dierks, T., Koenig, T. 2011. Resting-state EEG in schizophrenia: auditory verbal hallucinations are related to shortening of specific microstates. Clin. Neurophysiol. 122(6), 1179-1182. doi:10.1016/j.clinph.2010.10.042.

Klein, J.C., Rushworth, M.F.S., Behrens, T.E.J., Mackay, C.E., de Crespigny, A.J., D'Arceuil, H., JohansenBerg, H. 2010. Topography of connections between human prefrontal cortex and mediodorsal thalamus studied with diffusion tractography. Neurolmage 51(2), 555-564. doi:10.1016/j.neuroimage.2010.02.062.

Koenig, T., Lehmann, D., Merlo, M.C., Kochi, K., Hell, D., Koukkou, M. 1999. A deviant EEG brain microstate in acute, neuroleptic-naive schizophrenics at rest. Eur. Arch. Psychiatry Clin. Neurosci. 249(4), 205-211.

Koenig, T., Lehmann, D., Saito, N., Kuginuki, T., Kinoshita, T., Koukkou, M. 2001a. Decreased functional connectivity of EEG theta-frequency activity in first-episode, neuroleptic-naïve patients with schizophrenia: preliminary results. Schizophr. Res. 50(1-2), 55-60.

Koenig, T., Marti-Lopez, F., Valdes-Sosa, P. 2001b. Topographic time-frequency decomposition of the EEG. Neurolmage 14(2), 383-390. doi:10.1006/nimg.2001.0825.

Koenig, T., Prichep, L., Dierks, T., Hubl, D., Wahlund, L.O., John, E.R., Jelic, V. 2005a. Decreased EEG synchronization in Alzheimer's disease and mild cognitive impairment. Neurobiol. Aging 26(2), 165-171. doi:10.1016/j.neurobiolaging.2004.03.008.

Koenig, T., Prichep, L., Lehmann, D., Sosa, P.V., Braeker, E., Kleinlogel, H., Isenhart, R., John, E.R. 2002. Millisecond by millisecond, year by year: normative EEG microstates and developmental stages. Neurolmage 16(1), 41-48. doi:10.1006/nimg.2002.1070.

Koenig, T., Studer, D., Hubl, D., Melie, L., Strik, W.K. 2005b. Brain connectivity at different time-scales measured with EEG. Philos. Trans. R. Soc. Lond. B. Biol. Sci. 360(1457), 1015-1023. doi:10.1098/rstb.2005.1649.

Kottlow, M., Jann, K., Dierks, T., Koenig, T. 2012. Increased phase synchronization during continuous face integration measured simultaneously with EEG and fMRI. Clin. Neurophysiol. 123(8), 1536-1548. doi:10.1016/j.clinph.2011.12.019.

Laufs, H., Krakow, K., Sterzer, P., Eger, E., Beyerle, A., Salek-Haddadi, A., Kleinschmidt, A. 2003. Electroencephalographic signatures of attentional and cognitive default modes in spontaneous brain activity fluctuations at rest. Proc. Natl. Acad. Sci. U. S. A. 100(19), 11053-11058. doi:10.1073/pnas.1831638100. 
Lehmann, D., Faber, P.L., Galderisi, S., Herrmann, W.M., Kinoshita, T., Koukkou, M., Mucci, A., PascualMarqui, R.D., Saito, N., Wackermann, J., Winterer, G., Koenig, T. 2005. EEG microstate duration and syntax in acute, medication-naive, first-episode schizophrenia: a multi-center study. Psychiatry Res. 138(2), 141-156. doi:10.1016/j.pscychresns.2004.05.007.

Lehmann, D., Ozaki, H., Pal, I. 1987. EEG alpha map series: brain micro-states by space-oriented adaptive segmentation. Electroencephalogr. Clin. Neurophysiol. 67(3), 271-288.

Liu, Z., de Zwart, J.A., Chang, C., Duan, Q., van Gelderen, P., Duyn, J.H. 2014. Neuroelectrical decomposition of spontaneous brain activity measured with functional magnetic resonance imaging. Cereb. Cortex 24(11), 3080-3089. doi:10.1093/cercor/bht164.

Liu, Z., de Zwart, J.A., Yao, B., van Gelderen, P., Kuo, L.W., Duyn, J.H. 2012. Finding thalamic BOLD correlates to posterior alpha EEG. Neurolmage 63(3), 1060-1069. doi:10.1016/j.neuroimage.2012.08.025.

Lopes da Silva, F. 1991. Neural mechanisms underlying brain waves: from neural membranes to networks. Electroencephalogr. Clin. Neurophysiol. 79(2), 81-93.

Lopes da Silva, F.H., van Lierop, T.H., Schrijer, C.F., van Leeuwen, W.S. 1973. Organization of thalamic and cortical alpha rhythms: spectra and coherences. Electroencephalogr. Clin. Neurophysiol. 35(6), 627-639.

Lopes da Silva, F.H., Vos, J.E., Mooibroek, J., Van Rotterdam, A. 1980. Relative contributions of intracortical and thalamo-cortical processes in the generation of alpha rhythms, revealed by partial coherence analysis. Electroencephalogr. Clin. Neurophysiol. 50(5-6), 449-456.

Maldjian, J.A., Laurienti, P.J., Kraft, R.A., Burdette, J.H. 2003. An automated method for neuroanatomic and cytoarchitectonic atlas-based interrogation of fMRI data sets. Neurolmage 19(3), 1233-1239.

Mantini, D., Perrucci, M.G., Del Gratta, C., Romani, G.L., Corbetta, M. 2007. Electrophysiological signatures of resting state networks in the human brain. Proc. Natl. Acad. Sci. U. S. A. 104(32), 13170-13175. doi:10.1073/pnas.0700668104.

Musso, F., Brinkmeyer, J., Mobascher, A., Warbrick, T., Winterer, G. 2010. Spontaneous brain activity and EEG microstates. A novel EEG/fMRI analysis approach to explore resting-state networks. Neurolmage 52(4), 1149-1161. doi:10.1016/j.neuroimage.2010.01.093. 
Nair, A., Treiber, J.M., Shukla, D.K., Shih, P., Müller, R.A. 2013. Impaired thalamocortical connectivity in autism spectrum disorder: a study of functional and anatomical connectivity. Brain 136(Pt 6), 1942-1955. doi:10.1093/brain/awt079.

Nishida, K., Morishima, Y., Yoshimura, M., Isotani, T., Irisawa, S., Jann, K., Dierks, T., Strik, W., Kinoshita, T., Koenig, T. 2013. EEG microstates associated with salience and frontoparietal networks in frontotemporal dementia, schizophrenia and Alzheimer's disease. Clin. Neurophysiol. 124(6), 1106-1114. doi:10.1016/j.clinph.2013.01.005.

Pascual-Marqui, R.D., Michel, C.M., Lehmann, D. 1995. Segmentation of brain electrical activity into microstates: model estimation and validation. IEEE Trans. Biomed. Eng. 42(7), 658-665. doi: 10.1109/10.391164.

Raichle, M.E., MacLeod, A.M., Snyder, A.Z., Powers, W.J., Gusnard, D.A., Shulman, G.L. 2001. A default mode of brain function. Proc. Natl. Acad. Sci. U. S. A. 98(2), 676-682. doi:10.1073/pnas.98.2.676.

Reep, R.L., Corwin, J.V. 2009. Posterior parietal cortex as part of a neural network for directed attention in rats. Neurobiol. Learn. Mem. 91(2), 104-113. doi:10.1016/j.nlm.2008.08.010.

Ritter, P., Moosmann, M., Villringer, A. 2009. Rolandic alpha and beta EEG rhythms' strengths are inversely related to fMRI-BOLD signal in primary somatosensory and motor cortex. Hum. Brain Mapp. 30(4), 1168-1187. doi:10.1002/hbm.20585.

Schwab, S., Koenig, T., Federspiel, A., Dierks, T., Jann, K. 2013. Thalamic relay of frequency-specific EEG scalp field maps. Proc. Intl. Soc. Mag. Reson. Med. 21, 3246.

Sherman, S.M. 2007. The thalamus is more than just a relay. Curr. Opin. Neurobiol. 17(4), 417-422. doi:10.1016/j.conb.2007.07.003.

Smith, S.M., Fox, P.T., Miller, K.L., Glahn, D.C., Fox, P.M., Mackay, C.E., Filippini, N., Watkins, K.E., Toro, R., Laird, A.R., Beckmann, C.F. 2009. Correspondence of the brain's functional architecture during activation and rest. Proc. Natl. Acad. Sci. U. S. A. 106(31), 13040-13045. doi:10.1073/pnas.0905267106.

Stevens, A., Günther, W., Lutzenberger, W., Bartels, M., Müller, N. 1996. Abnormal topography of EEG microstates in Gilles de la Tourette syndrome. Eur. Arch. Psychiatry Clin. Neurosci. 246(6), 310-316. 
Strik, W.K., Chiaramonti, R., Muscas, G.C., Paganini, M., Mueller, T.J., Fallgatter, A.J., Versari, A., Zappoli, R. 1997. Decreased EEG microstate duration and anteriorisation of the brain electrical fields in mild and moderate dementia of the Alzheimer type. Psychiatry Res. 75(3), 183-191.

Tononi, G., Koch, C. 2008. The neural correlates of consciousness: an update. Ann. N. Y. Acad. Sci. 1124, 239-261. doi:10.1196/annals.1440.004.

Van de Ville, D., Britz, J., Michel, C.M. 2010. EEG microstate sequences in healthy humans at rest reveal scale-free dynamics. Proc. Natl. Acad. Sci. U. S. A. 107(42), 18179-18184. doi:10.1073/pnas.1007841107.

Whitfield-Gabrieli, S., Thermenos, H.W., Milanovic, S., Tsuang, M.T., Faraone, S.V., McCarley, R.W., Shenton, M.E., Green, A.I., Nieto-Castanon, A., LaViolette, P., Wojcik, J., Gabrieli, J.D.E., Seidman, L.J. 2009. Hyperactivity and hyperconnectivity of the default network in schizophrenia and in first-degree relatives of persons with schizophrenia. Proc. Natl. Acad. Sci. U. S. A. 106(4), 1279-1284. doi:10.1073/pnas.0809141106.

Yuan, H., Zotev, V., Phillips, R., Drevets, W.C., Bodurka, J. 2012. Spatiotemporal dynamics of the brain at rest-exploring EEG microstates as electrophysiological signatures of BOLD resting state networks. Neurolmage 60(4), 2062-2072. doi:10.1016/j.neuroimage.2012.02.031.

Zhang, D., Snyder, A.Z., Fox, M.D., Sansbury, M.W., Shimony, J.S., Raichle, M.E. 2008. Intrinsic functional relations between human cerebral cortex and thalamus. J. Neurophysiol. 100(4), 1740-1748. doi: $10.1152 /$ jn. 90463.2008 .

Zhang, D., Snyder, A.Z., Shimony, J.S., Fox, M.D., Raichle, M.E. 2010. Noninvasive functional and structural connectivity mapping of the human thalamocortical system. Cereb. Cortex 20(5), 1187-1194. doi: $10.1093 /$ cercor/bhp182.

Zhou, Y., Liang, M., Jiang, T., Tian, L., Liu, Y., Liu, Z., Liu, H., Kuang, F. 2007. Functional dysconnectivity of the dorsolateral prefrontal cortex in first-episode schizophrenia using resting-state fMRI. Neurosci. Lett. 417(3), 297-302. doi:10.1016/j.neulet.2007.02.081. 


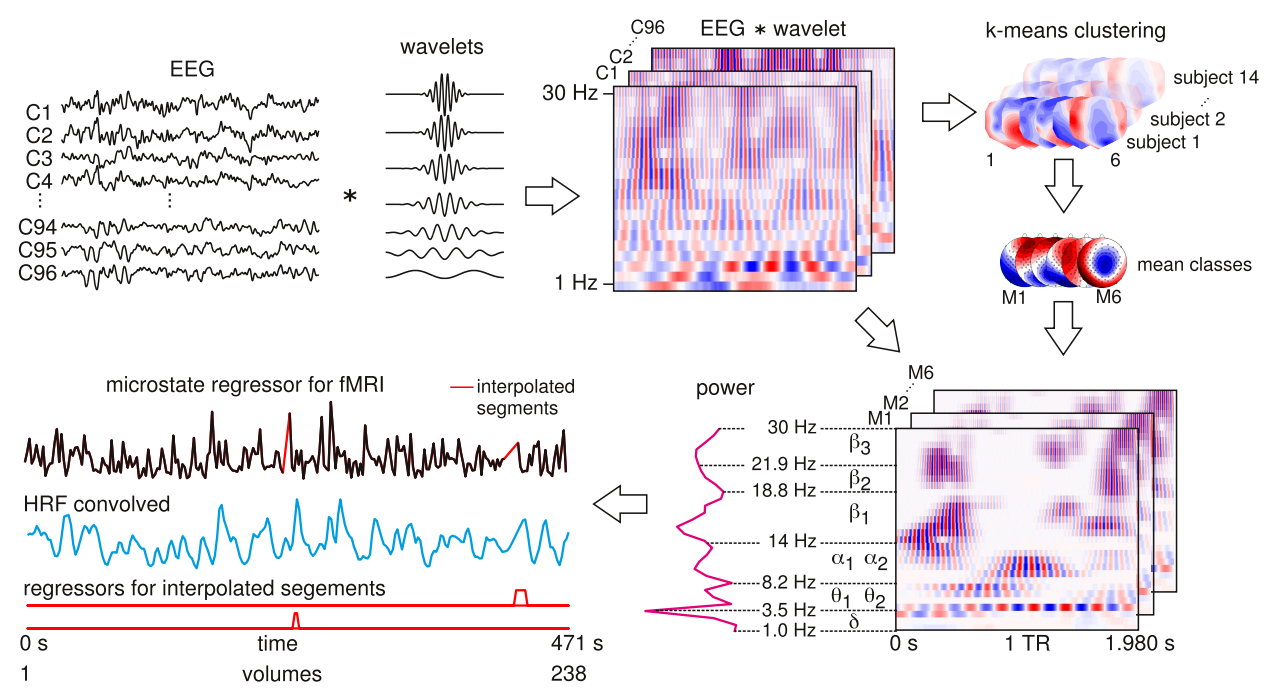

Figure 1. Steps involved in EEG microstate (MS) informed fMRI analysis. The EEG was subjected to a time-frequency analysis by convolution of the EEG with Gabor wavelets $(1-30 \mathrm{~Hz})$, resulting in a frequency $\times$ time $\times$ channel space. A k-means clustering algorithm yielded six prototypical topographic maps per participant, which were averaged into six group mean MS classes. Each topography vector of all EEG wavelet coefficients was then assigned to the best fitting mean MS class, and the time-frequency dynamics of each microstate class were reconstructed based on this assignment. For each fMRI TR, the power of the dynamics was averaged for the eight EEG standard frequency bands, resulting in MS regressor timeseries corresponding to the TRs, and timeseries were available the for eight standard frequency bands, and the six MS classes, resulting in a total of 48 regressors per subject. These regressors were convolved with the canonical HRF, and segments of low EEG data quality were modeled in the subsequent GLM. 

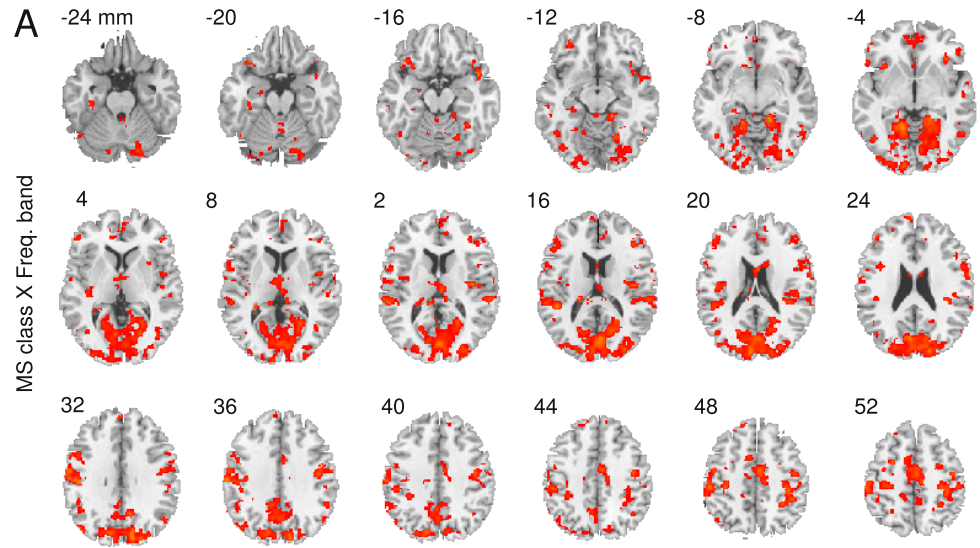

$16 \quad 20$
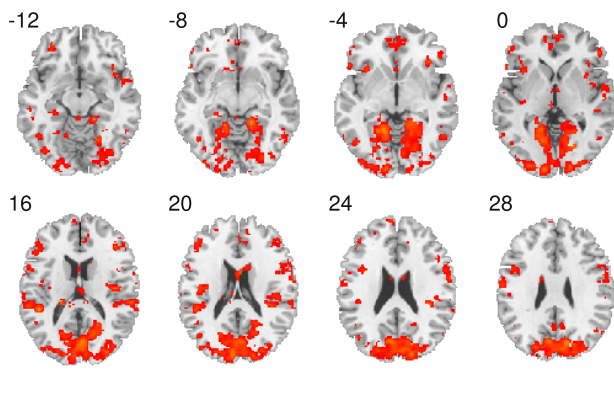

28
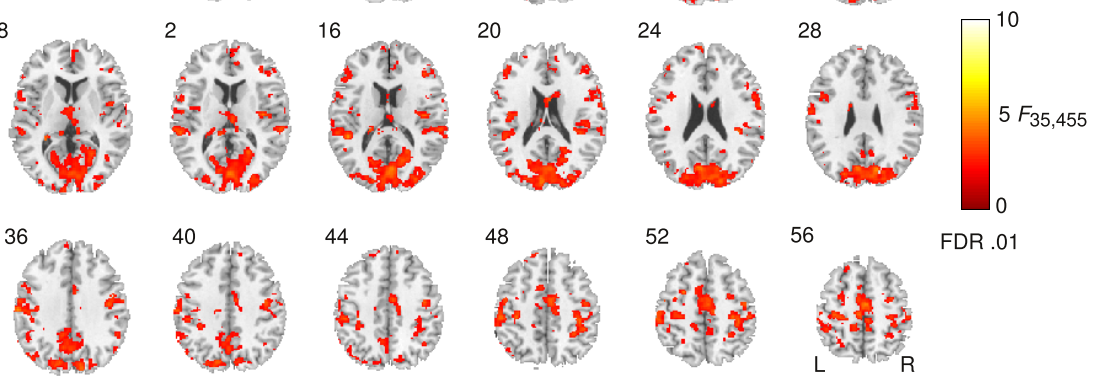

48
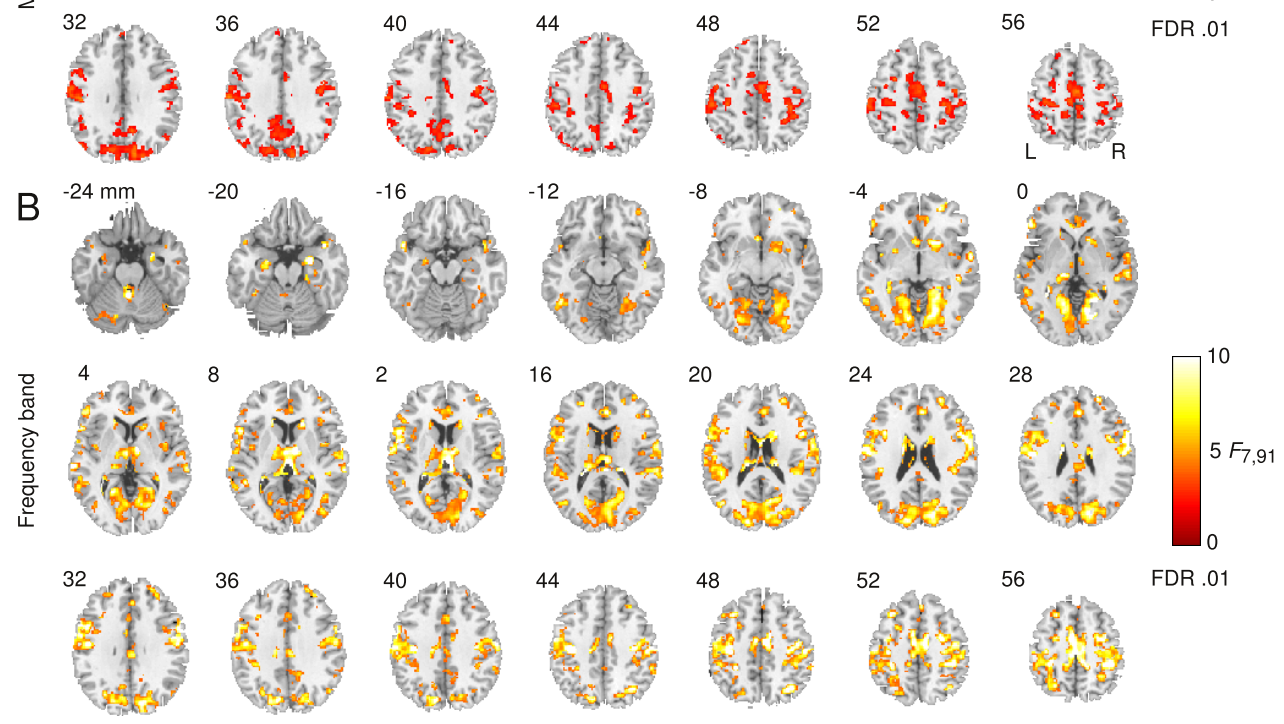

52

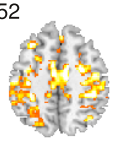

56
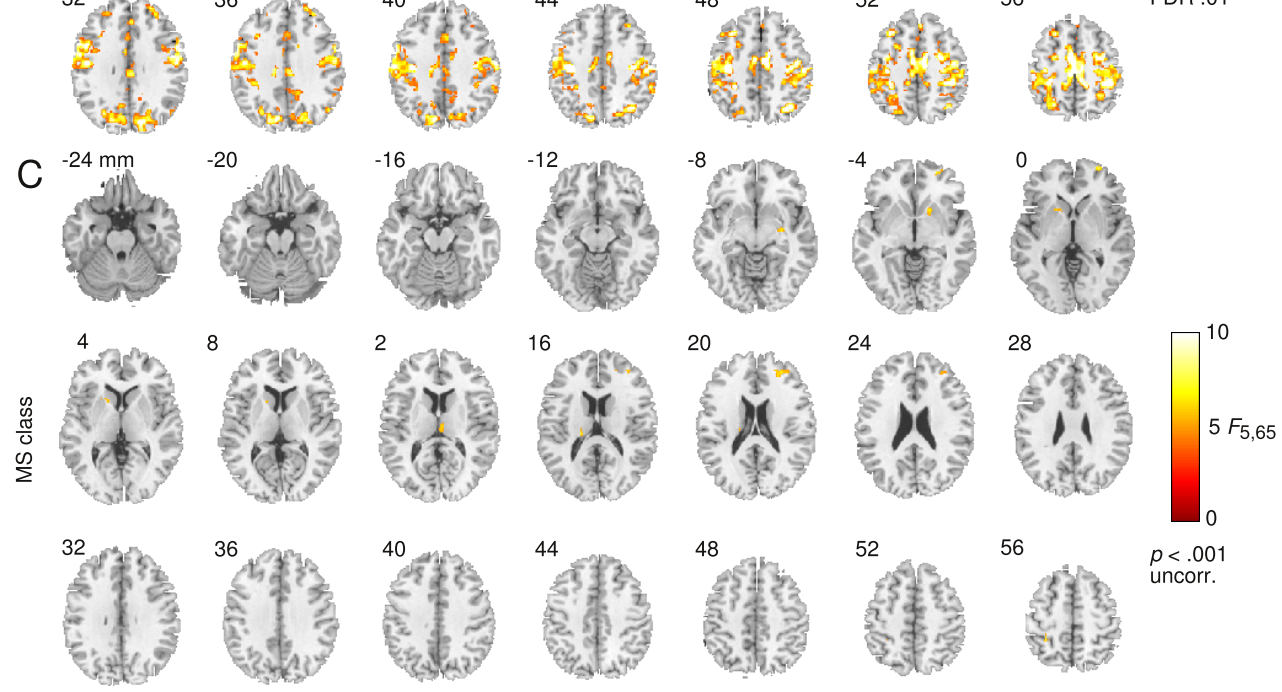

FDR .01

Figure 2. Microstate (MS) informed fMRI group analysis (14 participants). F-maps from random effects analysis of MS fluctuations in six MS classes and within eight standard EEG frequency bands $(6 \times 8$ ANOVA) to model fMRI beta estimates. These maps show distributed subcortical and cortical regions predicting BOLD activity depending on frequency band and/or MS class. All three maps show involvement of the thalamus, with Map B showing particular strong involvement. A) Voxels associated with both frequency band and MS class (interaction), peak voxel in the left paracentral lobule $(F=5.78 ;-6-2260 \mathrm{~mm})$. B) Regions associated with frequency changes only (main effect), peak voxel in the left postcentral gyrus ( $F=27.5 ;-36-1842 \mathrm{~mm}$ ). C) Regions associated with class changes only (main effect), peak voxel in the right superior orbitofrontal gyrus $(F=8.29 ; 3062-2 \mathrm{~mm})$. 


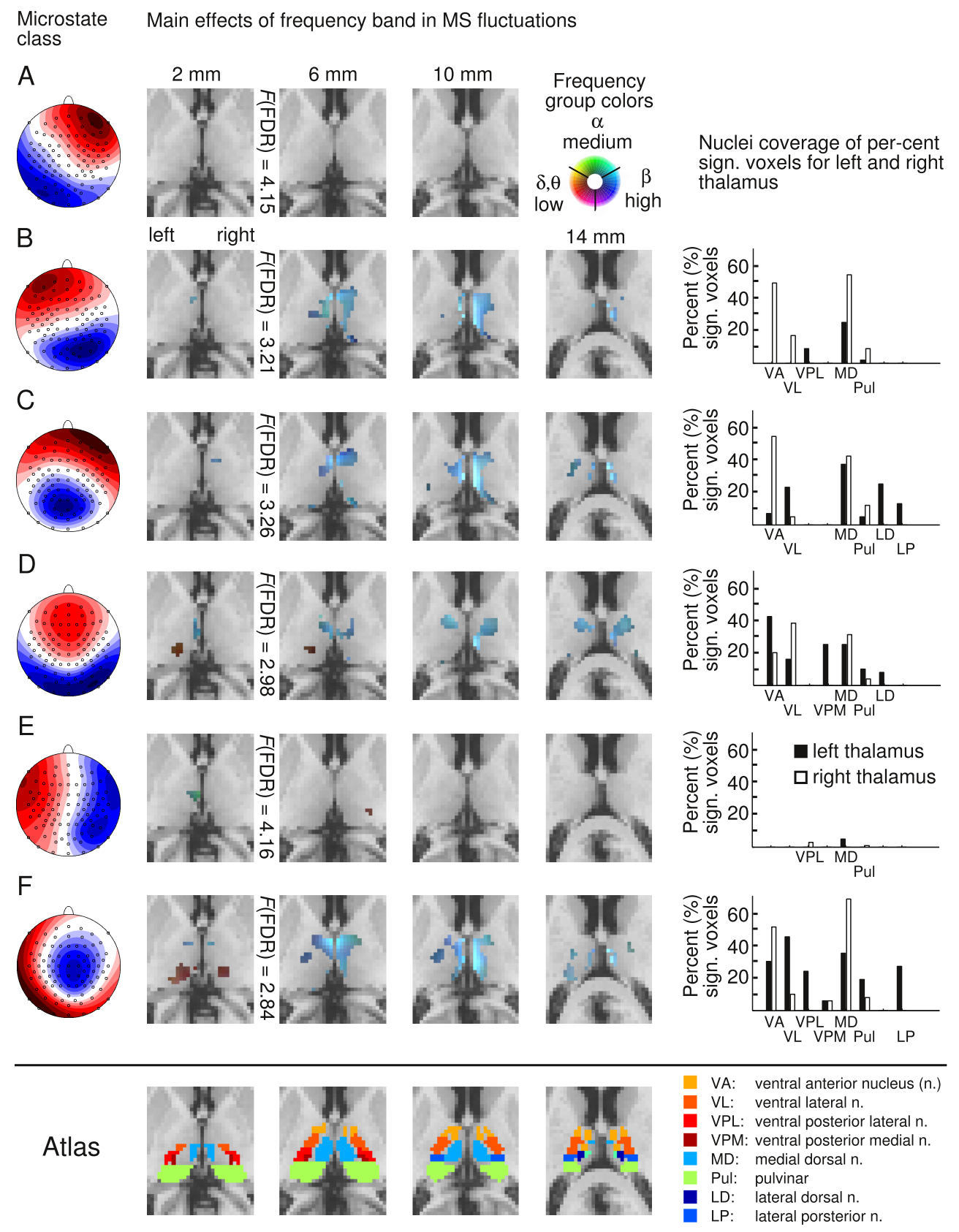

Figure 3. Six MS classes (A-F) and their corresponding thalamic areas associated with the main effect of frequency band in 14 participants. A voxel wise one-way ANOVA was conducted for each of the six MS classes, with the BOLD beta estimates as dependent variables and MS fluctuations along eight different standard EEG frequency bands as independent variables. Beta estimates of voxels with a significant $F$-value were categorized into three frequency groups and transferred into a RGB color values that reflected frequency group (low, medium or high). Colors reflect consistent thalamic activity to the low (red; $\delta$ and $\theta$ ), medium (green; $\alpha$ ), and high (blue; $\beta$ ) frequency bands across participants. For example, a mixed color such as cyan is associated with larger $\mathrm{fMRI}$ beta estimates in MS fluctuations within high and medium frequencies. There were no significant effects for MS class A. The atlas in the lower portion of the figure allows for comparison with common thalamic subdivisions; bar plots on the right show percentage coverage of significant voxels for eight thalamic nuclei. 


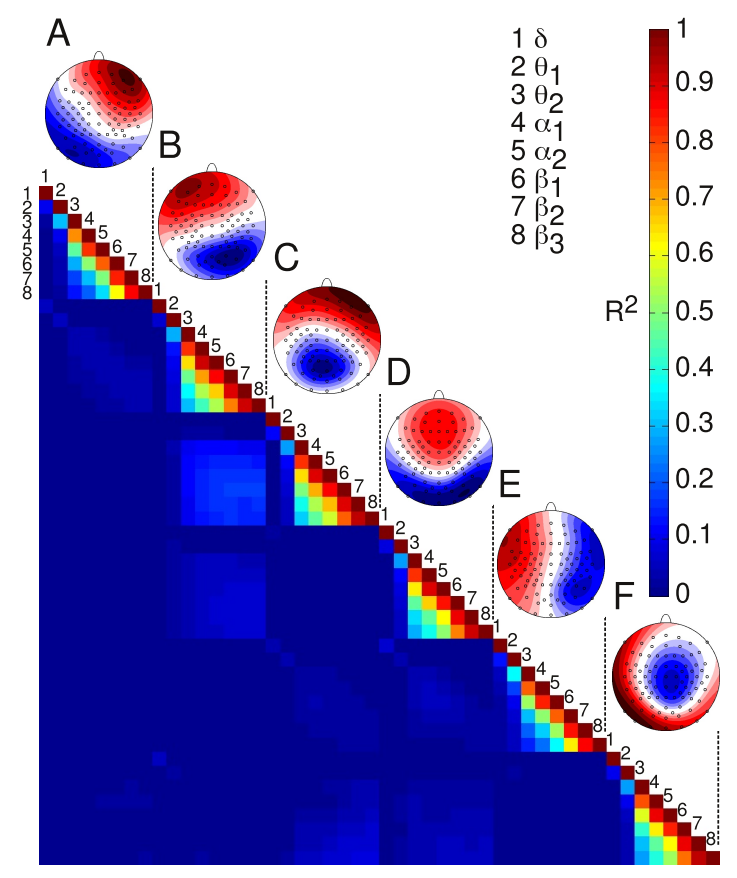

Figure 4. $R^{2}$ matrix of the 48 regressors to show the percentage shared variance of correlated regressors. Regressors are ordered according to the microstates $A-F$, and within the microstates according to the 8 frequency bands $\left(\delta-\beta_{3}\right)$. Regressors correlated only with their two adjacent regressors of the respective upper and lower frequency band, for example $\alpha_{1}$ correlated with $\theta_{2}$ and $\alpha_{2}$. Further, the two lowest frequency bands $\delta$ and $\theta_{1}$ did generally not correlate with any other regressors, and there was no correlation between microstate classes. 